\title{
Strates
}

STRATES Matériaux pour la recherche en sciences sociales

13 | 2007

Paysage urbain: genèse, représentations, enjeux contemporains

\section{Paolo Monti et le projet de Bologne}

Italo Zannier

\section{(2) OpenEdition}

Journals

Édition électronique

URL : http://journals.openedition.org/strates/6162

DOI : $10.4000 /$ strates.6162

ISSN : $1777-5442$

Éditeur

Laboratoire Ladyss

Édition imprimée

Date de publication : 31 décembre 2007

ISSN : 0768-8067

Référence électronique

Italo Zannier, « Paolo Monti et le projet de Bologne », Strates [En ligne], 13 | 2007, mis en ligne le 24 novembre 2008, consulté le 08 septembre 2020. URL : http://journals.openedition.org/strates/6162 ; DOI : https://doi.org/10.4000/strates.6162

Ce document a été généré automatiquement le 8 septembre 2020

Tous droits réservés 


\title{
Paolo Monti et le projet de Bologne
}

\author{
Italo Zannier
}

1 Paolo Monti (1908-1982) est toujours considéré comme un maître fondamental de la photographie italienne de l'après-guerre: auteur et théoricien en même temps qu'historien et enseignant.

2 Dans les années 1940, surtout durant son séjour à Venise alors qu'il était dirigeant d'industrie de 1945 à 1953, P. Monti encore photographe amateur a amorcé une intense recherche sur le langage photographique. Il se rallie et suit aussitôt les nouvelles orientations sémantiques proposées notamment par Otto Steinert, promoteur de la Subjektive Fotografie (1952), et par les Américains Aaron Siskind, Harry Callahan, Minor White, qu'il a plus tard aussi particulièrement étudiés et dans quelque mesure imités.

3 Né en 1908 à Novara dans le Piémont, P. Monti a principalement suivi des études d'économie, et a été diplômé de l'université Bocconi de Milan, sans cependant abandonner ses multiples intérêts culturels dans la sphère de l'art, et pas seulement de l'art figuratif.

4 Il trouva finalement dans la photographie la vocation artistique la plus conforme à sa personnalité, et en même temps la plus conceptuelle. Homme de culture raffiné, il se nourrit très vite du débat contemporain et des rencontres avec des écrivains, des peintres, et des historiens d'art, avec lesquels il s'associa souvent, jusque dans les projets photographiques. Il commença en 1953 en tant que professionnel, abandonnant définitivement le travail dans l'entreprise. Il fit aussitôt " école », spécialement parmi les jeunes photographes italiens (de Fulvio Roiter à Gianni Berengo Gardin) et dans les derniers mois de 1947 fonda à Venise le cercle La Gondola. La Gondola fut un centre significatif dans l'histoire de la photographie italienne de l'après-guerre, une sorte d'espace de médiation idéologique entre « néo-réalisme » et « formalisme ».

5 Par la suite, arrivé à Milan, il se consacra passionnément à l'enseignement auprès de la Scuola Umanitaria ${ }^{1}$ puis, de 1970 à 1973, au DAMS (Disciplines des arts, de la musique et du spectacle) de l'université de Bologne, département fondé entre autres par Umberto Eco, Tomàs Maldonaldo, Benedetto Marzullo. Au sein de l'université italienne, le DAMS était alors une formation de pointe sur les questions de l'art et de la 
communication. Là, il rencontre et fréquente des historiens et des chercheurs tels que Lucio Gambi et Andrea Emiliani ainsi que l'urbaniste Pierluigi Cervellati. C'est alors une période de grand bouillonnement culturel et politique qui l'enthousiasme, en particulier lorsque est émise l'hypothèse d'un programme de documentation photographique exhaustive et à grande échelle des monuments et des centres historiques de l'Émilie-Romagne. Ce travail commencera par le relevé phototopographique de Bologne.

6 P. Monti s'était entre-temps « spécialisé » dans la photographie d'architecture ; aussi le thème d'un relevé urbain rencontra-t-il sans aucun doute sa sensibilité culturelle et son talent figuratif. À Bologne, c'est un courageux programme, soutenu par l'administration municipale, qui fut à l'origine de la demande d'un relevé phototopographique. Ce programme prévoyait le sauvetage de la structure urbaine et citadine du centre historique, sauvetage non seulement architectural mais avant tout social, cherchant le plus possible à assurer la présence des habitants - artisans, retraités, des personnes le plus souvent âgées et économiquement faibles - dans les habitations d'origine familiale, maisons et appartements quelquefois délabrés qui devaient cependant être assainis correctement. Dans le centre historique, on se préoccupa de restaurer quand cela était possible, en installant en attendant les habitants dans des "édifices parkings", et de la sorte on put agir tout de suite, en entreprenant les travaux de réhabilitation. On confia donc à P. Monti la tâche de « documenter » ce territoire urbain dans sa situation actuelle, selon une démarche qui consentît de « congeler » l'image de la ville.

7 P. Monti choisit une forme de représentation par séquences, rue par rue du centre historique, selon des itinéraires les plus possibles organisés et rythmés, mais sans la circulation, et sans la pollution de la publicité et du stationnement, pour avoir « pour une fois au moins », dit-il alors, une image " propre » de la ville, lisible du trottoir aux toitures. Ce grand relevé photographique constitua en Italie un exemple pour d'autres interventions de documentation du paysage et des centres urbains; P. Monti fut en effet chargé d'appliquer cette méthode de travail à d'autres villes de l'Émilie-Romagne, notamment Modène et Cesena.

Beaucoup de ces images se retrouvèrent, outre dans les archives municipales et régionales, dans de nombreux livres de photographie qui représentent aujourd'hui un précieux condensé de l'image historique des villes et des petits bourgs de l'Émilie, un « document » irremplaçable, surtout si l'on pense à la transformation souvent sauvage advenue depuis lors.

9 Mais le fascinant projet de Bologne, projet-pilote du point de vue sociologique et urbanistique, après les restaurations et l'amélioration de la qualité de l'habitat dans les édifices provisoirement abandonnés par leurs vieux propriétaires placés dans des "parkings", ne mena pas aux résultats escomptés. Et souvent, vue la valeur économique atteinte, les quartiers furent au contraire affectés à des bureaux et à des locaux professionnels ; tandis que dans certains cas, les vieux propriétaires et locataires étaient entre-temps disparus et leurs héritiers se sentaient toujours moins intéressés, même du point de vue affectif.

Mais ce projet de Bologne - dont le maire était alors le Professeur Renato Zangheri - fut une utopie extraordinaire à laquelle prit part avec enthousiasme le photographe $\mathrm{P}$. Monti, offrant en tout état de cause une image inoubliable de la ville, même si celle-ci est transfigurée dans une atmosphère proche de la peinture métaphysique, dans le 
« silence » de ses images où n'apparaît aucun être humain, et semble carrément un lieu d'après l'apocalypse. P. Monti fut de toute façon fidèle jusqu'à la fin à cette "utopie " de la représentation photographique, voire topo-photographique, dont le modèle était la technique, rigoureusement archivistique, développée au $\mathrm{xIX}^{\mathrm{e}}$ siècle par les photographes florentins Alinari : maison par maison, monument par monument, église par église, rue par rue...

11 Ce fut même au prix d'un renoncement, avec une grande modestie, à sa créativité visuelle inépuisable, à laquelle il donnait en revanche libre cours dans l'enceinte de son atelier, où il a systématiquement insisté sur la recherche d'un langage photographique, recherche qui le place au sommet de l'histoire européenne de l'après-guerre, bien que cet auteur reste à découvrir au niveau européen et mondial, et non seulement en Italie où il est toujours considéré comme un maître fondamental de la culture photographique.

12 Le fonds photographique P. Monti renferme principalement une documentation assez étendue d'architecture, ancienne comme moderne, et du paysage italien, des régions du Nord à celles du Sud, consultables également dans un catalogue ad hoc, mais difficile à trouver. À cet ensemble de photographies d'architecture, d'art et d'urbanisme, s'ajoute un corpus représentatif et fondamental de photographies relevant davantage d'une démarche de création personnelle, notamment celles que P. Monti a qualifiées d'«abstractions involontaires", recherches de formes et de structures graphiques, souvent sans appareil photographique, dans lesquelles l'auteur a tenté d'exprimer avec force son angoisse créative et artistique.

Traduction Hélène Jannière

13 Nota bene : Les photographies de Paolo Monti ne sont pas en ligne. Ceux qui souhaitent les voir peuvent se reporter à l'édition papier du Strates $n^{\circ} 13$ qu'ils pourront acheter en écrivant au Ladyss, 2 rue Valette, 75005 Paris.

Note sur le projet de Bologne (Hélène Jannière)

Les photographies de Bologne ici présentées s'inscrivent dans une vaste campagne de relevés des centres villes d'Emilie Romagne, amorcée sous l'impulsion de l'historien d'art et conservateur en chef des Monuments historiques, Andrea Emiliani. Associée à des relevés dessinés en vue d'une classification typologique du bâti, une campagne photographique de grande ampleur a été conduite durant l'été 1969, sous la direction de l'architecte urbaniste Pierluigi Cervellati ${ }^{2}$ et d'Andrea Emiliani ${ }^{3}$. Les 5000 photographies de Paolo Monti, photographe alors déjà célèbre et appelé pour Bologne par A. Emiliani à la suite de son travail sur les Apennins, avaient un but documentaire et entraient dans un processus de projet, fondé sur une analyse minutieuse des architectures et des typologies, de conservation du centre médiéval, renaissant et baroque considéré « comme un tout unitaire ». À Bologne, les études pour le Plan régulateur du centre historique venaient à peine de s'achever, provoquant des polémiques sur la conservation « extensive » de la ville ancienne : les auteurs tentaient de convaincre qu'une restauration bien conduite ne pouvait s'appliquer uniquement aux monuments historiques, mais allait des monuments à l'architecture mineure, des façades à la conservation des typologies architecturales, des modes de construction aux enduits ${ }^{4}$. Car cette opération ne recouvrait pas uniquement la réhabilitation des structures bâties. Elle tentait de trouver une réponse au développement incontrôlé de la ville : de 1961 à 1971, la population du centre historique n'avait cessé de diminuer et la tendance était à l'expansion de la périphérie urbaine, en même temps qu'à la 
dépopulation des collines, de la montagne et de la plaine. Parallèlement, la spéculation immobilière et la construction de bureaux tendaient à combler dans le centre historique les vides laissés par les bombardements de la seconde guerre mondiale ${ }^{5}$.

Mais, au-delà de la conservation des structures physiques, la restauration du centre de Bologne se voulait une « intervention sur le centre historique qui assure la permanence des classes sociales et des activités productives existantes aujourd'hui ${ }^{6} "$, refusant toute opération de conservation par la restauration en dehors de la « conservation sociale». Les fonds habituellement consacrés à la construction de logements sociaux en périphérie furent alloués à la réhabilitation des habitations du centre ville et au maintien sur place de leurs occupants, tentant d'endiguer ainsi le phénomène de relégation des couches modestes en banlieue. Cette opération ne fut possible que par la volonté politique de trouver un autre modèle de développement, et de fonder l'intervention architecturale et urbaine sur une analyse précise de l'existant. Analyse typologique et morphologique du bâti, relevé métrique, recherches dans les cadastres historiques, enquêtes sur la composition démographique et sociale de la population et, enfin, relevé photographique sont les instruments d'analyse qui ont permis une connaissance fine du tissu urbain et de ses typologies. Le Piano per la costruzione economica e popolare (Plan pour la construction économique et populaire, PEEP), adopté le 7 mars 1973, intègre le résultat de ces enquêtes en vue de l'acquisition de terrains par la municipalité et du sauvetage du patrimoine.

16 Au regard de la notion de paysage urbain, les intérêts de l'opération de Bologne sont multiples.

17 Elle concrétise pour partie la réflexion sur la notion de «paysage urbain » qui a eu lieu en Italie depuis le début du siècle et surtout depuis le milieu des années 1950 : à cette date, le débat architectural et urbain italien se fait l'écho de la notion britannique de townscape. Parallèlement, les premiers résultats de la Reconstruction dans une Italie en plein «miracle économique " se traduisent par des modernisations parfois brutales (éventrements, élargissements de voies, destructions) et commencent à alerter les milieux d'intellectuels et d'artistes. Les congrès de l'Institut national des urbanistes (INU) de 1957 à Lucques (« Défense et valorisation du paysage urbain et rural») et celui de 1959 à Lecce (" Le visage de la ville ») ont confirmé la relation déjà présente en Italie, entre la notion de paysage urbain, la sauvegarde et la conservation. En outre, ces deux congrès ont débattu l'extension de la notion de sauvegarde du monument au paysage.

18 La notion de paysage urbain ne prend pas uniquement en compte la conservation de l'édifice historique, mais tente de considérer le continuum du bâti :

Le territoire historique ainsi défini et délimité, ayant une valeur historique et artistique définie, est considéré comme un monument unique pour l'homogénéité de ses valeurs matérielles et socioculturelles. [...] Le centre historique est ainsi défini comme un organisme urbain unitaire et non comme un ensemble de bâtiments ${ }^{7}$.

19 La photographie de P. Monti documente les particularités architecturales, mais révèle en même temps l'image de ce continuum construit, qui dessine le paysage urbain bolognais :

Ce qui nous a le plus surpris, durant l'opération « documentation globale » a été la continuité du tissu architectural, le rythme ininterrompu, exaltant, des portiques ; la compacité des environnements et des espaces [...]. Loin de tout soupçon de vouloir geler, c'est-à-dire muséifier la ville, nous pouvons dire que la documentation de cet été-là démontre que, à peine possible, nous pourrions 
entreprendre même un travail de restauration, de couture, de neutralisation des ruptures qu'a quelques fois provoquées la spéculation immobilière avec la complaisance de certaines autorités ${ }^{8}$.

L'opération de Bologne connaît un important écho tout d'abord en Italie, où elle devient un exemple pour d'autres communes (Brescia, Modène). N'étant plus exclusivement dans une logique spatiale, elle rompt avec la logique du Piano regolatore generale (PRG) ${ }^{9}$. Au-delà, l'opération reçoit en 1974 les éloges du Conseil de l'Europe et de l'Unesco. En France au début des années 1970, les revues professionnelles lui assurent une réception certaine, en écho à la contestation de la spéculation immobilière et des rénovations urbaines alors en plein essor dans les grandes villes françaises. Enfin, l'ouvrage de P. Cervellati ${ }^{10}$ relatant l'expérience de Bologne et en tirant des éléments de méthode pour la conservation est traduit aux éditions du Seuil en 1981, sous le titre de La nouvelle culture urbaine.

1 L'opération de Bologne devient ainsi un exemple à la fois de luttes urbaines et de méthode de conservation "intégrée », c'est-à-dire maintien sur place d'une population économiquement fragile et maintien de la structure du tissu urbain mineur. En témoigne l'ouvrage paru en 1976 au Centre de recherche d'urbanisme, Les ensembles historiques et l'urbanisme ${ }^{11}$, qui met au centre de sa problématique la notion de "conservation intégrée » à partir de l'exemple de Bologne, en relation avec la définition du paysage urbain.

En somme, l'opération de Bologne illustre la forte articulation, en Italie entre paysage urbain et définition du patrimoine historique d'une ville. Elle montre le rôle joué par la photographie pour révéler les structures de ce patrimoine historique - du monument aux édifices « courants », à la répétition typologique.

\section{NOTES}

1. Les écoles Umanitaria sont issues de la société homonyme (Società Umanitaria) fondée en 1893, qui promeut dans les années 1920 les Biennales de Monza, devenues en 1930 les Triennales de Milan. Ces écoles sont consacrées aux arts décoratifs et appliqués (NdT).

2. P. Cervellati devient adjoint du maire (R. Zangheri) chargé de l'urbanisme en 1970.

3. Les résultats de la première phase de la campagne furent exposés de mai à juillet 1970 à Bologne, Palais d'Accursio et publiés : P. L. Cervellati, A. Emiliani, R. Renzi, R. Scannavini, Bologna centro storico, Catalogo del Piano per il Centro Storico elaborato dal comune di Bologna, Bologne, Alfa edizioni, 1970, 286 p.

4. Voir P. Cervellati, « Il censimento fotografico dei centri storici dell'Emilia e della Romagna ", in Istituto per i beni artistici culturali e naturali della Regione Emilia Romagna, Paolo Monti e l'età dei piani regolatori (1960-1980), Catalogue d'exposition, Bologne, Alfa edizioni, 1983, p. 15. 
5. E. Barbiani, G. Conti, Politiques urbaines et luttes sociales à Bologne. Reconstruction, « miracle italien » et crise dans une " municipalité rouge », t. I, Paris, CNRS, Centre de sociologie urbaine, DGRST, 1979, p. 277.

6. P. Cervellati, R. Scannavini, Bologna : Politica e metodologia del restauro nei centri storici, Bologne, Il Mulino, 1975, p. 93.

7. P. Cervellati, R. Scannavini, op. cit., p. 93.

8. P. Cervellati, « Il censimento fotografico », Il Giorno, 19 septembre 1969, cité par

A. Emiliani, Dal Museo al territorio, Bologne, Alfa edizioni, 1974.

9. E. Barbiani, G. Conti, op. cit.

10. Édition originale : P. Cervellati, R Scannavini, op. cit.

11. W. Ostrowski, Les ensembles historiques et l'urbanisme, Paris, CRU, 1976, 373 p.

\section{AUTEUR}

\section{ITALO ZANNIER}

Historien de la photographie, Professeur à l'université des Lettres de Venise, département d'Histoire de l'art. 